\title{
Adaptation and extinction of stimulus control in children from two socioeconomic levels
}

\author{
JEAN L. BRESNAHAN \\ Herbert H. Lehman College, Bronx, New York 10468 \\ and \\ MARTIN M. SHAPIRO and JOHN C. MILAZZO \\ Emory University, Atlanta, Georgia 30322
}

\begin{abstract}
Sixty 4-year-old children from two socioeconomic levels were run in a three-phase experiment designed to investigate the effect of Phase 1 training upon the acquisition of stimulus control by two stimulus dimensions. Phase 1 consisted of three different training conditions; one stimulus dimension was correlated with reinforcement, and the second stimulus dimension was: (a) also correlated with reinforcement, (b) uncorrelated with reinforcement, or (c) held constant. In Phase 2, all subjects received reinforcement correlated with both dimensions. In Phase 3, the two dimensions were tested conjunctively, disjunctively, and separately. The results showed that stimulus control was more dependent upon Phase 1 training in children from the higher socioeconomic level and was more dependent upon a priori preferences in children from the lower socioeconomic level.
\end{abstract}

Kamin (1968, 1969), employing a conditioned suppression procedure with rats, found that prior training to one element of a compound noise-light conditioned stimulus effectively blocked conditioning to the other element, provided the unconditioned stimulus remained unchanged.

Other examples of blocking in animals have been observed. Vom Saal and Jenkins (1970; , by prior discrimination training to red vs. green, recorded the failure of tone vs. noise to exhibit stimulus control when both stimulus dimensions subsequently were superimposed and both predicted reinforcement. In an attempt to further study the processes involved in blocking, a number of animal researchers (Mackintosh \& Honig, 1970; Thomas, Freeman, Svinicki, Burr, \& Lyons, 1970) have determined that preliminary discrimination training on one stimulus dimension can enhance or inhibit the control acquired by a second stimulus dimension, as measured by the generalization gradient. The results have been consistent with a selective attention hypothesis to explain blocking insofar as prior discrimination training along one dimension was observed to affect the control available to other dimensions.

Trabasso and Bower (1968), studying college students, used geometric shapes and/or dots near the shapes as relevant stimuli. Three conditions were studied. Subjects in Condition 1 received training with both the dot and shape present, and both positively correlated with reinforcement. Subjects in Condition 2 received training with both stimuli present, but only one correlated with reinforcement. Subjects in Condition 3 received training with either the shape or the dot, the other stimulus being absent (or constant). In the second phase, in all three conditions, both stimuli were positively correlated with reinforcement; and each stimulus was then tested separately to determine the control, if any, it had acquired. The results were clear. In Condition 1, the subjects were split as to whether they used shape, dot, or both stimuli as solutions. In Condition 2, all subjects blocked the second stimulus, and in Condition 3, almost all subjects exhibited blocking. Prior training to one stimulus, where the second stimulus was either absent or uncorrelated with reinforcement, had a much different effect than prior training where both stimuli could be used as solutions.

Lyczak and Tighe (1975) attempted to test the extension of this phenomenon to children and found only weak evidence of blocking. Bresnahan, Smith, and Shapiro (Note 1), studying 4-year-old children, found significant blocking in children from a lower socioeconomic level but not in children from a higher socioeconomic level. In Phase 1, one stimulus element was reinforced and a second stimulus dimension was held constant on each trial, a procedure similar to Condition 3 used by Trabasso and Bower (1968). In Phase 2, the second stimulus was reinforced on all trials; the originally reinforced stimulus was available and reinforced on either $100 \%, 75 \%$, or $50 \%$ of the trials and held constant on the remaining trials. In Phase 3, the two elements were tested conjunctively, disjunctively, and separately. Lower socioeconomic subjects: (a) performed well on the first element and blocked the second element if the first element was available on $100 \%$ of the Phase 2 trials, (b) performed poorly on both elements if the first 
element was available on $75 \%$ of the Phase 2 trials, and (c) performed poorly on the first element and well on the second element if the first element was available on $50 \%$ of the Phase 2 trials. Higher socioeconomic subjects performed well on both elements under all conditions; however, predominant control passed from the first element to the second element after the availability of the first element had been reduced in Phase 2 . These results coincided with the results from the various animal studies which demonstrated that the degree of blocking decreased as prevailing reinforcement dependency was progressively altered. These results also coincided with the results obtained by Trabasso and Bower (1968) in their Condition 3. A very important restriction was that significant blocking occurred only in children from a lower socioeconomic level. The children from a higher socioeconomic level did not display the blocking found in either animals or college students, a finding that is difficult to interpret. The result is, however, possibly similar to a related finding that performance on incidental learning tasks may not be a monotonic function of age (Siegel, 1968; Siegel \& Stevenson, 1966).

The purpose of the present experiment was to replicate the general procedure of the three conditions employed by Trabasso and Bower (1968) with children from higher and lower socioeconomic levels.

\section{METHOD}

\section{Subjects}

Sixty preschool children from two day care centers were used as subjects. Thirty higher socioeconomic children were sampled from private day care centers and $\mathbf{3 0}$ lower socioeconomic children were sampled from publicly supported day care centers. The mean ages were 4.36 years and 4.53 years, respectively.

The children were randomly assigned to three experimental groups of 20 subjects each, 10 subjects from each socioeconomic level.

\section{Apparatus}

The apparatus, previously described in detail (Bresnahan, 1966), was positioned on a small table facing the subject. It consisted of a panel $61 \mathrm{~cm}$ high and $75.5 \mathrm{~cm}$ wide, with an opening of $15 \times 32 \mathrm{~cm}$ at the bottom. Resting on the table within this opening was a blue tray extending $14 \mathrm{~cm}$ in front of the panel. Its back was $4 \mathrm{~cm}$ high and flush with the panel. On the left-hand side of this first panel, two 9-cm-square cartoon faces were inserted, one directly above the other. The top face was a smiling one; the bottom face was a sad one with tears. Each face could be illuminated.

Attached to the right-hand side of this first panel was a second panel, $25.4 \mathrm{~cm}$ high and $38.1 \mathrm{~cm}$ wide, on which was mounted a green chute, $12 \mathrm{~cm}$ wide and $9.5 \mathrm{~cm}$ high, which protruded from the panel at a 45-deg angle. The entire apparatus had $3-\mathrm{cm}$ diagonal red-and-white stripes painted on it. The objects used as stimuli were two buttons with a diam of $1.7 \mathrm{~cm}$, one yellow and one green, and two buttons with a diam of $1.2 \mathrm{~cm}$, one yellow and one green. Except for size and color, the buttons were equal in all respects and had the appearance of standard shirt buttons.

A $6-\mathrm{V}$ bell and a $6-\mathrm{V}$ buzzer could be activated manually by the experimenter.

\section{Procedure}

The subjects were tested individually in one experimental session lasting approximately $20 \mathrm{~min}$. There was no visual contact between the experimenter and the subject because of the height of the main panel of the apparatus. All subjects were given the following instructions which were modified from those used by Bresnahan (1966): "Now we're going to play a game. In this blue tray (pointing) there will be two things like this (experimenter dropping two practice stimuli in the tray). One of them is right; the other is wrong. I want you to pick up the one you think is right and put it in the green chute here (pointing). If you put the right one in the chute, this happy face (pointing) will light up and it will sound like this (demonstration of light and bell). If you put in the wrong one, this sad face (pointing) will light up and it will sound like this (demonstration of light and buzzer). Now you put one in just for practice. (After subject places either of the two stimuli in the chute. experimenter presses the "wrong" pushbutton). That one was wrong. When you pick up the wrong one, then go ahead and put the other one in. (After subject places the other one in chute, the "right" pushbutton is pressed.) You understand how to play now, don't you? You want the happy face to come on as many times as you can; you don't want the sad face to come on. You want to get as many right as you can. All right, we'll begin now."

The experiment was divided into three phases for each of three experimental conditions within each socioeconomic level. The three conditions differed only in the first phase. In Condition 1, Phase 1, the subject was presented the small yellow (SY) button and the large green (LG) button for 12 trials. In Condition 2, Phase 1, the subject was presented six trials of the SY and LG buttons and six trials of the large yellow (LY) and small green (SG) buttons in an unsystematic order. In Condition 3, Phase 1, one-half of the subjects was presented with 12 trials of the SY and LY buttons, and one-half of the subjects. was presented with 12 trials of the SY and SG buttons. The reinforcement dependency was counterbalanced within each condition; for one-half of the subjects, size was the solution stimulus, and for the other one-half of the subjects, color was the solution stimulus. The reinforced value of the size dimension was the smaller of the two buttons. The reinforced value of the color dimension was yellow. The subjects in Condition 1, in choosing SY over LG, could have selected according to size, or color, or both. In this condition, the two stimuli were redundant in the first phase. The experimental treatment of the counterbalanced subgroups of Condition 1 was, therefore, identical; the counterbalanced subgroups served as a purely statistical control. In the other two conditions, there was a unique correct stimulus value. In Condition 2, the second dimension was uncorrelated with reinforcement (i.e., $50 \%$ reinforcement); in Condition 3 , the second dimension was held constant.

In Phase 2, for all three conditions, the same two buttons, SY and LG, were presented for all 12 trials. The SY button was always "correct." The subject had, therefore, as solution possibilities, small, or yellow, or both. This phase was the same as Phase 1 for Condition 1.

All subjects received 24 acquisition trials without any interruption between Phases 1 and 2. On each trial, the subjects could hear the experimenter tossing the buttons into the tray; the location of the stimuli in the tray was not a variable. Before each trial began, the lid on the tray was closed; it was raised by the experimenter as soon as the buttons were tossed into the tray. Immediately after the correct button was placed in the chute, the lid was closed again and the procedure was repeated.

In the third phase, designated as the test phase, all subjects were given a total of 48 trials presented in an unsystematic sequence of 12 trials, each of four types of stimulus pairs; (1) a SY and a LG button, the two "correct" stimulus values being conjunctive, (b) a LY and a SG button, the two "correct" stimulus values being disjunctive, (c) a SY and a SG button, both being the same size, and (d) a SY and a LY button, both being the same color. The choice of the subject was noted for each test trial. 
After the last trial of Phase 2, and immediately before the test phase, each subject was told: "Now see if you can guess which button is right without the light, bell, or buzzer coming on. Go ahead and put one button in the chute just like before. If you do well here, I will give you a toy.

The two buttons (one of the four types of test trials) were then presented in the usual manner, and after the subject had dropped one button into the chute, the lid was closed, the remaining button was removed, and the next test pair was presented. No feedback was given to the subject concerning the correctness of his choice.

\section{RESULTS}

The test phase had consisted of four types of choice trials: (a) SY vs. LG, (b) LY vs. SG, (c) SY vs. SG, and (d) SY vs. LY. Three analyses of variance were computed: one for the 12 SY-LG trials, one for the 12 LY-SG trials, and a mixed design including both the 12 SY-SG and the 12 SY-LY trials.

The results from the SY-LG test trials, in which the two stimulus dimensions were conjunctive, did not yield a significant difference between the number of correct choices by the higher socioeconomic subjects $(88 \%)$ and the lower socioeconomic subjects $(90 \%)$. There were neither differences among the three conditions $(92 \%, 82 \%$, and $91 \%$, respectively), nor an interaction between socioeconomic level and condition. There was a significant effect of the originally trained stimulus dimension, $F(1,48)=$ $10.34, \mathrm{p}<.01$. Preference for SY was greater when size $(98 \%)$ had been the solution in Phase 1 than when color $(79 \%)$ had been the solution in Phase 1 . No effect of condition was expected because both solutions (size and color) were available to the subject in the choice of SY. Subjects who had attended to size, color, or both, would have made the same choice. Any differences among conditions would have been the result of differences in the amount of original learning. A difference in original learning was reflected in the fact that subjects originally trained on size did better than subjects originally trained on color.

On the second type of test trial, LY-SG, the data represent the number of times the subject selected the stimulus value which had been correlated with reinforcement in Phase 1 (small or yellow). For example, if the choice of size (small) was reinforced in Phase 1 , then the score indicated the number of times, out of 12 , SG was chosen over LY. If color (yellow) was reinforced in Phase 1, then the score reflected the number of times LY was chosen over SG. There were no overall significant effects due to condition $(48 \%, 70 \%$, and $74 \%$, respectively), socioeconomic level (higher $=69 \%$, lower $=58 \%$ ), or their interactions. A significant difference was obtained between stimuli, $F(1,48)=7.74, p<.01$; there was more preference for size after Phase 1 training with size $(\mathbf{7 8 \%})$ than for color after Phase 1 training with color $(50 \%)$.
An examination of the total for each of the three conditions suggested a possible difference between Condition 1 and the other two conditions. A significant difference was found between Condition 1 and Conditions 2 plus 3 combined, $F(1,48)=4.66$, $\mathrm{p}<.05$. This difference was produced by the fact that some subjects in Condition 1 preferred the originally trained stimulus, and some subjects in Condition 1 preferred the second stimulus. In Condition 1, the designation "originally trained stimulus" was arbitrarily made by the experimenter and was nonfunctional for the subject.

The data from the SY-SG and SY-LY test trials reflect the two types of trials on which stimuli were constant along one dimension; therefore, the two types of trials were combined into one analysis. No significant overall effects were observed due to conditions, stimuli, socioeconomic levels, or any interactions of these variables. When the data of Conditions 2 and 3 were compared within each socioeconomic level, a significant interaction was obtained, $F(1,48)=4.32, \quad p<.05$. The lower socioeconomic level subjects in Condition 3 performed more reliably $(\mathbf{8 1 \% )}$ than the higher level subjects in Condition $3(72 \%)$, but the lower level subjects in Condition 2 performed less reliably $(65 \%)$ than the higher level subjects in Condition $2(83 \%)$.

The only significant main effect was due to the difference in performance on the first-trained stimulus and the second-trained stimulus, $F(1,48)=$ $6.55, p<.05$. This effect was a direct measure of the difference in stimulus control between the stimulus correlated with reinforcement in both Phases 1 and 2 $(82 \%)$, and the other stimulus correlated with reinforcement only in Phase 2 (69\%). Phase 2 acquisition of the second stimulus dimension was reduced by the Phase 1 training. This was the blocking effect.

The triple interaction of First-Second Trained Stimulus by Phase 1 Stimulus by Socioeconomic Level was also significant, $F(1,48)=4.30, p<.05$. In general, the higher socioeconomic level subjects who were first trained on color responded relatively poorly to size $(62 \%)$, whereas the lower level subjects who were first trained on color did better on size $(78 \%)$. The lower level subjects who were first trained on size, however, responded poorly to color $(59 \%)$ whereas the higher level subjects who were first trained on size responded better on color $(77 \%)$. Both socioeconomic levels did better on the originally trained stimulus if it had been size (higher $=92 \%$, lower $=89 \%$ ) than if it had been color $(80 \%$ and $68 \%$, respectively).

The percentage of correct responses for each condition are shown in Table 1 for both the test trials on which the first-trained stimulus was tested separately and the test trials on which the second-trained stimulus was tested separately. 
Table 1

Percentage of Correct Responses for Separate Test Trials of the Two Stimulus Dimensions

\begin{tabular}{|c|c|c|c|c|}
\hline \multirow{2}{*}{$\begin{array}{l}\text { Phase } 1 \text { Training } \\
\text { Stimulus Tested } \\
\text { (First or Second } \\
\text { Trained) }\end{array}$} & \multicolumn{2}{|c|}{ Yellow } & \multicolumn{2}{|c|}{ Small } \\
\hline & $\begin{array}{l}\text { Yellow } \\
\text { (First) }\end{array}$ & $\begin{array}{l}\text { Small } \\
\text { (Second) }\end{array}$ & $\begin{array}{l}\text { Small } \\
\text { (First) } \\
\end{array}$ & $\begin{array}{l}\text { Yellow } \\
\text { (Second }\end{array}$ \\
\hline & \multicolumn{4}{|c|}{ Higher Socioeconomic Group } \\
\hline $\begin{array}{l}\text { Condition } 1 \\
\text { Condition } 2 \\
\text { Condition } 3\end{array}$ & $\begin{array}{l}80 \\
83 \\
77\end{array}$ & $\begin{array}{l}83 \\
58 * \\
43^{*}\end{array}$ & $\begin{array}{r}80 \\
100 \\
95\end{array}$ & $\begin{array}{l}67 \\
92 \\
73\end{array}$ \\
\hline & \multicolumn{4}{|c|}{ Lower Socioeconomic Group } \\
\hline $\begin{array}{l}\text { Condition } 1 \\
\text { Condition } 2 \\
\text { Condition } 3\end{array}$ & $\begin{array}{l}73 \\
48^{*} \\
83\end{array}$ & $\begin{array}{l}70 \\
70 \\
93\end{array}$ & $\begin{array}{r}83 \\
83 \\
100\end{array}$ & $\begin{array}{l}72 \\
58^{*} \\
48^{*}\end{array}$ \\
\hline
\end{tabular}

*Chance performance within $95 \%$ confidence interval.

\section{DISCUSSION}

The results will be discussed in reference to the three experimental conditions.

In Condition 1, subjects from both socioeconomic levels performed well on test trials on which the two stimulus dimensions were tested separately. These subjects had received continuous training on small yellow vs. Iarge green and had clearly learned to select the stimulus on the basis of either small, or yellow, or both. The results from the test trials on which the subjects were forced to choose between the two stimulus values showed that some subjects chose small and that other subjects chose yellow, small being preferred more often than yellow. Condition 1 may be viewed as a baseline or control procedure in which nondifferential training for size and color resulted in children from both socioeconomic levels learning both stimulus values.

In Condition 2, the subjects from the two socioeconomic levels performed quite dissimilarly. Phase 1 consisted of differential reinforcement of the two stimulus values in which one stimulus value was $100 \%$ reinforced and the other stimulus value was $50 \%$ reinforced (i.e., uncorrelated with reinforcement). Subjects from the higher socioeconomic level performed well on both stimulus values if small had been $100 \%$ reinforced in Phase 1 , but performed at a chance level on separate tests of size $(95 \%$ confidence level) if color had been $100 \%$ reinforced in Phase 1 . It could be hypothesized that the subjects initially tend to adopt a size hypothesis in their problem solving and that this hypothesis is rejected and not readopted if size is initially uncorrelated with reinforcement. Acquisition of size in Phase 2 is subsequently blocked. If size is initially $100 \%$ reinforced, the size hypothesis is maintained in Phase 1, and the color hypothesis is also adopted in Phase 2, when small and yellow become perfectly correlated with each other as well as perfectly correlated with reinforcement.

In Condition 2, subjects from the lower socioeconomic level performed well on size and at a chance level on color, independently of which stimulus had been reinforced in Phase 1. If size had been $100 \%$ reinforced in Phase 1 , they apparently stayed with size through Phase 2. If color had been $100 \%$ reinforced in Phase 1 , they either never learned color, or adopted and maintained a partially correct solution of size in Phase 1 (see Bresnahan \& Shapiro, 1972), and then stayed with size through Phase 2. Their behavior was a product of the initial preference for size and was not changed by the differential reinforcement of color in Phase 1 .

In Condition 3, one stimulus value was $100 \%$ reinforced, and the other stimulus value was held constant during Phase 1 training. The second stimulus was held constant at the value that was to be reinforced in Phase 2 (yellow or small). Subjects from the higher socioeconomic level responded similarly to the higher socioeconomic level subjects in Condition 2 . The second stimulus was blocked if it was small but not if it was yellow. This result is, however, different from that obtained by Bresnahan et al. (1975). In Phase 1 they held the second stimulus value constant within each trial, but varied it from trial to trial; this procedure did not produce blocking in higher socioeconomic level children.

In Condition 3, the behavior of the lower socioeconomic level children differed from their behavior in Condition 2 . In Condition 3, these subjects again responded correctly to size and blocked color if it had been held constant in Phase 1; however, the subjects for whom size had been held constant in Phase 1, learned both color and size prior to testing. The dimension held constant in Phase 1 could not be used as even an imperfect solution. Although size was readily learned in Phase 2 when it became available, color had been acquired in Phase 1 .

In general, attention in subjects from a higher socioeconomic level is controlled by the interaction of reinforcement dependencies and hypothesis testing during training. A hypothesis which is adopted and not confirmed is rejected and replaced by a new hypothesis. A hypothesis which has a high probability of adoption but is not relevant to the problem (held constant within and between trials) is also rejected and replaced by a new hypothesis. Attention in subjects from a lower socioeconomic level is controlled more by an a priori preference for a specific hypothesis. The preferred hypothesis is not discarded and attention to the related stimulus values is not blocked.

\section{NOTE}

1. Bresnahan, J. L., Smith, M. A., \& Shapiro, M. M. Blocking in children from different socioeconomic levels. Paper delivered at the convention of the Eastern Psychological Association, April 4, 1975, New York, New York.

\section{REFERENCES}

Bresnahan, J. L. The effect of task and incentive on concept acquisition with children from two socioeconomic levels (Doctoral dissertation, Emory University, 1966). Dissertation Abstracts International, 1967, 27, 2886B (University Microfilms No. 67-762).

Bresnahan, J. L.. \& Shapiro, M. M. Learning strategies in children from different socioeconomic levels. In $\mathrm{H}$. W. Reese (Ed.), Advances in child development and behavior. New York: Academic Press, 1972.

KAMIN, L. J. "Attention-like" processes in classical conditioning. In M. R. Jones (Ed.), Miumi symposium on the prediction of behavior: Aversive stimulation. Miami: University of Miami Press, 1968.

KAMIN, L. J. Selective association and conditioning. In N. J. Mackintosh \& W. K. Honig (Eds.), Fundamental issues in associative learning. Halifax: Dalhousi University Press, 1969.

Lyczak, R., \& Tighe, T. Stimulus control in children under a blocking paradigm. Child Development, 1975, 46, 115-122.

Mackintosh, N. J., \& Honig, W. K. Blocking and enhancement of stimulus control in pigeons. Journal of Comparative and Physiological Psychology, 1970, 73, 78-85.

SIEgEL, A. W. Variables affecting incidental learning in children. Child Development, 1968, 39, 957-968.

Siegel, A. W., \& Stevenson, H. W. Incidental learning: A developmental study. Child Development, 1966, 37, 811-817.

Thomas, D. R., Freeman, F., Svinicki, J. G., Burr, D. E. S., \& LyoNs, J. Effects of extra-dimensional training on stimulus generalization. Journal of Experimental Psychology Monograph, 1970, 83 (1, Part 2).

Trabasso, T., \& Bower, G. H. What is learned. In T. Trabasso \& G. H. Bower (Eds.), Attention in learning: Theory and research. New York: Wiley, 1968.

Vom SAal, W., \& Jenkins, H. M. Blocking the development of stimulus control. Learning and Motivation, 1970, 1, 52-64. 